\title{
Familial hemophagocytic lymphohistiocytosis
}

\author{
S. Raka $\cdot$ P. Nayar $\cdot$ R. Godbole $\cdot$ R. Manchanda
}

Received: 22 December 2008 / Accepted: 8 June 2009

(C) Indian Society of Hematology and Transfusion Medicine 2009

\begin{abstract}
A 45-day-old infant presented with hepatosplenomegaly and fever since 15 days. Hemogram revealed bicytopenia and bone marrow aspirate showed hemophagocytosis. With the history of death of two siblings, the baby was diagnosed with hemophagocytic lymphohistiocytosis (HLH), likely to be of familial origin.
\end{abstract}

Keywords Bone marrow $\cdot$ Cytopenia $\cdot$ Ferritin

S. Raka $\cdot$ P. Nayar $\cdot$ R. Godbole $\cdot$ R. Manchanda

Department of Pathology,

King Edward Memorial Hospital,

Pune, India

R. Manchanda $(\bowtie)$

E-mail:rman@vsnl.com

\section{Introduction}

Hemophagocytic lymphohistiocytosis (HLH), whether familial or acquired, is a rare condition characterized by highly stimulated but ineffective immune response that threatens the life of the patient and may lead to death unless arrested by appropriate treatment. The pathologic hallmark of the syndrome is aggressive proliferation of macrophages and histiocytes $[1,2]$.

In HLH-94, the first prospective international study for $\mathrm{HLH}$, the diagnosis of HLH was based on five criteria - fever, splenomegaly, bicytopenia, hemophagocytosis, hypertriglyceridemia and/or hypofibrinogenemia. In HLH-2004, three additional criteria were introduced; low/absent NK cell activity, hyperferritinemia and high soluble interleukin-2 receptor levels. Altogether five of these eight criteria should be fulfilled, unless history or molecular diagnosis is consistent with HLH [3].

\section{Case report}

A one-and-a-half month old female infant, third product of a nonconsanginuous marriage, presented with abdominal distension, generalized pallor and icterus. There was hepatomegaly of $2 \mathrm{~cm}$ and splenomegaly of $3 \mathrm{~cm}$.

On investigation, the child had $\mathrm{Hb}-8.9 \mathrm{~g} \%$, WBC $17,600 / \mathrm{cmm}$, and platelet count $-89000 / \mathrm{cmm}$. PBS showed a normocytic normochromic picture. Few neutrophils showed toxic granules. Platelets were low on smear. Bone marrow aspiration showed normoblastic erythropoiesis. Myeloid series showed mild toxic changes. There was an increase in histiocytes and lymphocytes with hemophagocytosis. Some of the histiocytes showed pigment which was positive for prussian blue stain. Further lab work-up showed:

Plasma fibrinogen - $60 \mathrm{mg} \%$

Bilirubin (total) - $6.1 \mathrm{mg} \%$ 


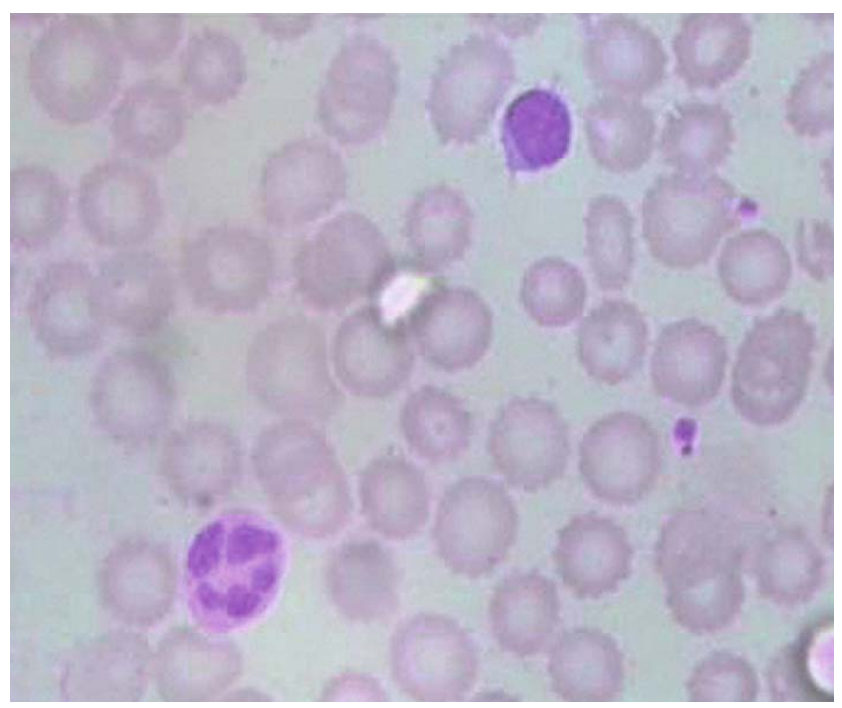

Fig. 1 Peripheral blood smear: normocytic normochromic RBCs with thrombocytopenia: (Leishman; 100X)

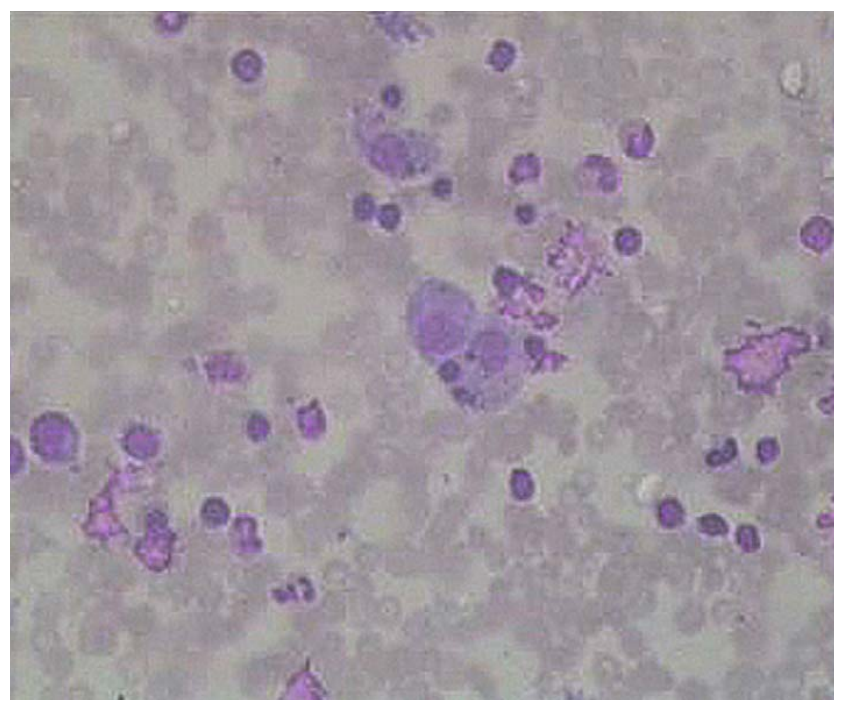

Fig. 2 Bone marrow aspirate: increase in lymphocytes and histiocytes with hemophagocytosis: (Leishman; 40X)

$$
\begin{aligned}
& \text { (Direct) - } 3.5 \mathrm{mg} \% \\
& \text { SGOT - } 35 \mathrm{IU} / \mathrm{L} \\
& \mathrm{SGPT}-36 \mathrm{IU} / \mathrm{L} \\
& \text { Serum ferritin - >1200 ng/ml } \\
& \text { Serum triglycerides - } 110 \mathrm{mg} \%
\end{aligned}
$$

The tests for viral infections, Koch's and TORCH were all negative. Further history revealed death of two siblings at the same age with similar complaints. Unfortunately they were not worked-up.

\section{Discussion}

HLH comprises familial (primary) hemophagocytic lymphohistiocytosis (FHL) and secondary HLH (SHLH), both

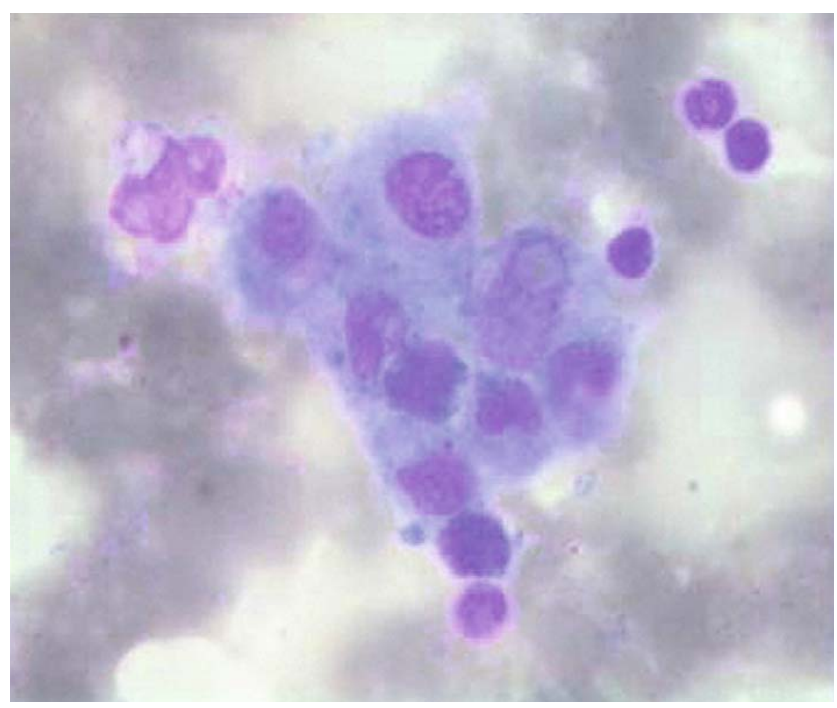

Fig. 3 Bone marrow aspirate: histiocytic cell showing hemophagocytosis and pigment: (Leishman; 100X)

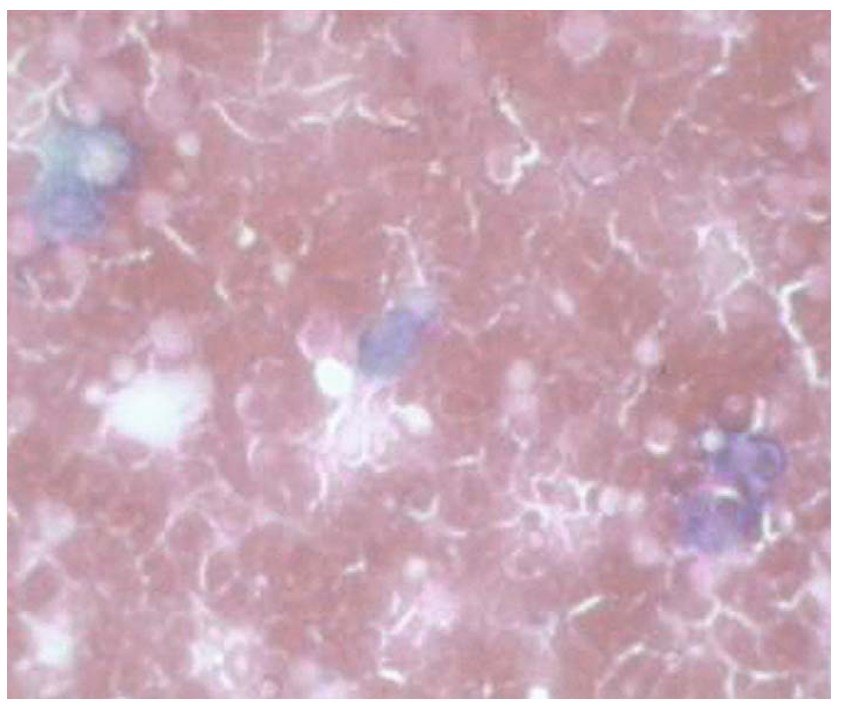

Fig. 4 Bone marrow aspirate: pigment stained with perls stain: (Perls stain; 100X)

clinically characterized by fever, hepatosplenomegaly, and cytopenias [4].

The clinical picture is due to increased inflammatory response caused by hyper secretion of pro-inflammatory cytokines [2]. The diagnosis of familial hemophagocytic lymphohistiocytosis is based on clinical criteria and confirmed by molecular testing [1].

Our case presented with cardinal features of HLH, i.e. fever, splenomegaly and bicytopenia. Bone marrow aspiration showed hemophagocytic lymphohistiocytosis. On work-up there was increased serum ferritin, decreased plasma fibrinogen and increased serum bilirubin. Confirmation by molecular studies was not possible. All the possible secondary causes were ruled out.

Above all, the age of presentation and history of death of two siblings with similar complaints supported the diagnosis 
of genetic HLH. Thus with proper history, routine biochemical and hematological work-up the diagnosis of hemophagocytic lymphohistiocytosis of familial origin was made.

\section{References}

1. Bhattacharya M, Ghosh MK (2008) Hemophagocytic lymphohistiocytosis - recent concept. J Assoc Physicians India $56: 453-457$
2. Janka G, Stadt UZ (2005) Familial and acquired hemophagocytic lymhohistiocytosis. American Society of Hematology Education Book 82-87

3. Henter JI, Horne A, Arico M et al. (2007) HLH-2004 Diagnostic and therapeutic guidelines for HLH. Pediatric Blood Cancer 48(2):124-131

4. Henter JI, Samuelson HA, Arico M et al. (2002) Treatment of HLH with HLH-94 immunochemotherapy and bone marrow transplantation. Blood 100(7):2367-2373 